\title{
High-Efficiency p-i-n Photodetectors on Selective-Area-Grown Ge for Monolithic Integration
}

\author{
Hyun-Yong Yu, Shen Ren, Woo Shik Jung, Ali K. Okyay, David A. B. Miller, and Krishna C. Saraswat, Fellow, IEEE
}

\begin{abstract}
We demonstrate normal incidence p-i-n photodiodes on selective-area-grown Ge using multiple hydrogen annealing for heteroepitaxy for the purpose of monolithic integration. An enhanced efficiency in the near-infrared regime and the absorption edge shifting to longer wavelength is achieved due to $0.14 \%$ residual tensile strain in the selective-area-grown Ge. The responsivities at $1.48,1.525$, and $1.55 \mu \mathrm{m}$ are $0.8,0.7$, and $0.64 \mathrm{~A} / \mathrm{W}$, respectively, without an optimal antireflection coating. These results are promising toward monolithically integrated on-chip optical links and in telecommunications.
\end{abstract}

Index Terms-Germanium, photodiode, selective, strain, tensile.

\section{INTRODUCTION}

$\mathbf{S}$ I-BASED devices for optical applications have been widely researched. However, a Si photodetector that operates in the $1.3-1.55-\mu \mathrm{m}$ wavelength range is a challenging task because of its relatively large indirect $(\sim 1.1 \mathrm{eV})$ and direct $(\sim 3.4 \mathrm{eV})$ bandgap energies. Since Ge naturally has a smaller direct bandgap energy of $0.8 \mathrm{eV}$, corresponding to $\sim 1.55-\mu \mathrm{m}$ wavelength, it is, however, a strong candidate for this application. Moreover, Ge is easy to integrate with the existing Si CMOS technology, further making it an attractive material for optical applications.

In particular, Ge optical detectors on $\mathrm{Si}$ are being aggressively researched as a potential solution for optoelectronic integration application. As a result, several heteroepitaxial techniques have been introduced to grow $\mathrm{Ge}$ on Si. For example, employing superlattice buffer layers to grow Ge layers effectively reduced the large lattice mismatch between $\mathrm{Si}$ and $\mathrm{Ge}$, yielding optical detectors with a quantum efficiency of $40 \%$ at $1.3 \mu \mathrm{m} \mathrm{[1].} \mathrm{Cyclic} \mathrm{thermal} \mathrm{annealing} \mathrm{is} \mathrm{another} \mathrm{method}$ of heteroepitaxial growth, which reported a responsivity of $0.56 \mathrm{~A} / \mathrm{W}$ at $1.55-\mu \mathrm{m}$ wavelength on $1-\mu \mathrm{m}$-thick Ge films grown on $\mathrm{Si}$ [2]. Moreover, molecular beam epitaxy is yet another method to grow $\mathrm{Ge}$ on $\mathrm{Si}$, and it can create thin strainrelaxed buffers on silicon substrate. This method showed an external quantum efficiency of $2.8 \%$ at $1.55 \mu \mathrm{m}$ [3]. These methods are focused on the bulk heteroepitaxial growth on $\mathrm{Si}$.

Manuscript received August 9, 2009. First published October 2, 2009; current version published October 23, 2009. This work was supported in part by FCRP Interconnect Focus Center. The review of this letter was arranged by Editor P. K.-L. Yu.

H.-Y. Yu, S. Ren, W. S. Jung, D. A. B. Miller, and K. C. Saraswat are with the Department of Electrical Engineering, Stanford University, Stanford, CA 94305 USA (e-mail: yuhykr@stanford.edu).

A. K. Okyay is with the Department of Electrical Engineering, Bilkent University, Ankara TR-06800, Turkey.

Color versions of one or more of the figures in this letter are available online at http://ieeexplore.ieee.org.

Digital Object Identifier 10.1109/LED.2009.2030905

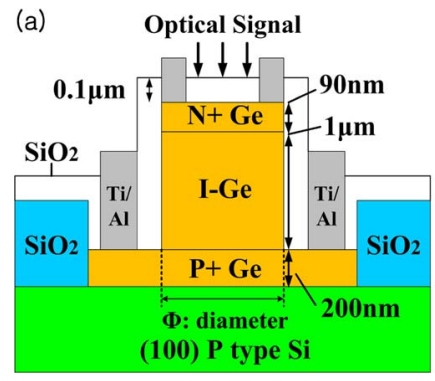

(b)

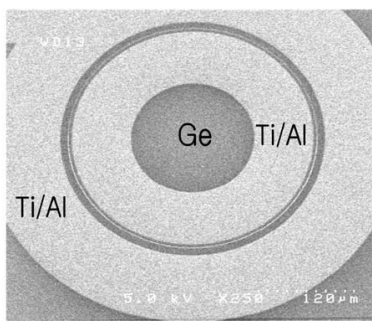

Fig. 1. (a) Schematic diagram of the cross section of normal incidence $\mathrm{Ge} / \mathrm{Si}$ p-i-n photodiode. (b) Scanning electron micrograph of the circular mesas of the photodiodes. The circular $\mathrm{p}$-i-n device has the ring-shaped contact with a ring width of $50 \mu \mathrm{m}$.

However, selective-area heteroepitaxy is a promising approach for the monolithic integration of Ge-based optoelectronics on Si CMOS VLSI platform and thus needs to be thoroughly studied. Several integration methods based on selective-area heteroepitaxy have been suggested for monolithic integration with waveguide. An evanescently coupled Ge waveguide photodetector demonstrates a responsivity of $0.89 \mathrm{~A} / \mathrm{W}$ at $1550 \mathrm{~nm}$ and a dark current density of $25 \mathrm{~mA} / \mathrm{cm}^{2}$ at $-1 \mathrm{~V}$ [4]. A butt-coupled Ge photodetector integrated in SOI rib waveguide showed a responsivity as high as $1 \mathrm{~A} / \mathrm{W}$ at a wavelength of $1550 \mathrm{~nm}$ and a low dark current density of $60 \mathrm{~mA} / \mathrm{cm}^{2}$ [5].

In this letter, we demonstrate a normal incidence $p-i-n \mathrm{Ge}$ photodiode by selectively growing Ge through patterned $\mathrm{SiO}_{2}$ on $\mathrm{Si}$ using the multiple hydrogen annealing for heteroepitaxy (MHAH) technique. We report a high-efficiency p-i-n photodiode with a high responsivity over a broad detection spectrum, making monolithically integrated on-chip or chip-tochip optical links more feasible.

\section{EXPERIMENT}

Fig. 1(a) shows the schematic cross section of a Ge $p-i-n$ photodiode. A 500-nm-thick $\mathrm{SiO}_{2}$ film was thermally grown on a lightly doped p-type (100) Si substrate at $1100{ }^{\circ} \mathrm{C}$. The $\mathrm{SiO}_{2}$ film was then patterned by a combination of dry etching followed by wet etching to define the desired locations for $\mathrm{Ge}$ growth. Ge epitaxial layers in a $p-i-n$ structure were selectively grown directly on $\mathrm{Si}$ in windows opened through the $\mathrm{SiO}_{2}$ layer. To obtain abrupt junctions and good electrical contact, a 200-nm-thick heavily in situ boron-doped Ge layer was deposited initially [6] at $400{ }^{\circ} \mathrm{C}$ and $8 \mathrm{~Pa}$. This was followed by annealing for $30 \mathrm{~min}$ at $800{ }^{\circ} \mathrm{C}$ in $\mathrm{H}_{2}$ ambient. The growth temperature was then increased to $600{ }^{\circ} \mathrm{C}$ for the formation of 1- $\mu \mathrm{m}$-thick intrinsic Ge layer, followed by another $800{ }^{\circ} \mathrm{C}$ hydrogen anneal. Finally, a heavily doped 90 -nm-thick 


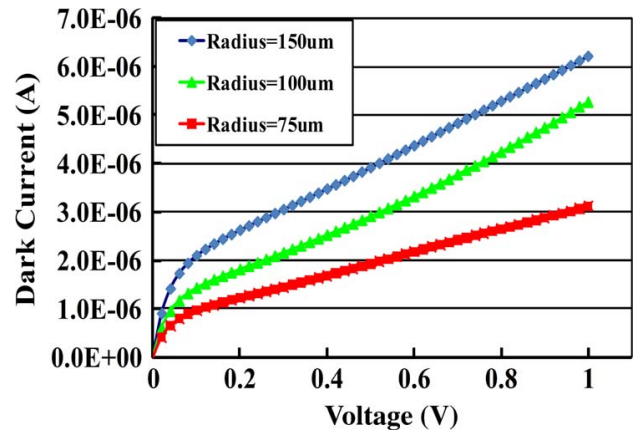

Fig. 2. Dark current versus reverse-bias curves of the circular-shaped mesa $\mathrm{Ge} / \mathrm{Si}$ p-i-n photodiodes.

$\mathrm{n}^{+}$-type Ge layer was grown at $600{ }^{\circ} \mathrm{C}$. To avoid recombination in the $\mathrm{n}^{+}$Ge layer, we needed a shallow $\mathrm{n}^{+}$layer and an abrupt $\mathrm{n}^{+} / i$ junction. To achieve this with a high level of n-type dopant activation, this layer was in situ doped with diluted $1 \%$ phosphine. The resultant $\mathrm{n}^{+}$junction has a depth of $97 \mathrm{~nm}$ and a peak electrically activated concentration of $2 \times 10^{19} \mathrm{~cm}^{-3}$. Because fast n-type dopant diffusion during the high-temperature process makes it difficult to fabricate $\mathrm{p}-\mathrm{i}-\mathrm{n}$ structure in Ge photodiodes, we fabricated the topmost $\mathrm{n}^{+}$layer without post hydrogen annealing to prevent phosphorus from diffusing inside the $1-\mu \mathrm{m}$-thick intrinsic Ge layer [7]. The rootmean-square roughness of the resulting film was determined to be $\sim 0.67 \mathrm{~nm}$ by $10 \times 10 \mu \mathrm{m}^{2}$ area atomic force microscopy scans [8], [9]. The samples annealed in nitrogen at $825{ }^{\circ} \mathrm{C}$ exhibited no reduction in surface roughness. Due to the selective heteroepitaxy and hydrogen annealing, the Ge layer had a low threading dislocation density count of $1 \times 10^{7} \mathrm{~cm}^{-2}$ based on the plan-view TEM, which is suitable for optoelectronic applications [10]. The $p-i-n$ Ge photodiodes were realized as mesa structures from $150-$ to $300-\mu \mathrm{m}$ diameter. The mesa structures were patterned by $\mathrm{HBr} / \mathrm{Cl}_{2}$ reactive ion etching of the Ge layer to a depth of $1 \mu \mathrm{m}$. On top of this Ge film, a 100-nm-thick low-temperature silicon oxide layer was deposited at $300{ }^{\circ} \mathrm{C}$ for surface passivation. Windows for the contacts were then patterned in the oxide and etched in HF, followed by metal deposition using electron-beam metal evaporation and photoresist liftoff. About $25 \mathrm{~nm}$ of $\mathrm{Ti}$ was used as contact material, topped with $\sim 45 \mathrm{~nm}$ of Au. Fig. 1(b) shows the SEM top view of the resulting $p-i-n$ Ge photodiode.

\section{RESUlTS AND Discussion}

The dark current density of the photodiode not only indicates the material quality but also determines the optical receiver sensitivity [11], [12]. Fig. 2 shows the dark current-voltage $I-V$ characteristics of photodiodes with various mesa radii. For large photodiodes with a radius of $150 \mu \mathrm{m}$, the dark current density is $9.9 \mathrm{q}$ at a reverse bias of $1 \mathrm{~V}$. The dark current can be related to bulk dark current density $\left(J_{\text {bulk }}\right)$ and the peripheral surface leakage density $\left(J_{\text {surf }}\right)$ through

$$
I_{\mathrm{dark}}=J_{\mathrm{bulk}} \text { Area }+J_{\mathrm{surf}} B \sqrt{\text { Area }}
$$

where $B$ is the $\sqrt{4 \pi}$ for a circular photodiode.

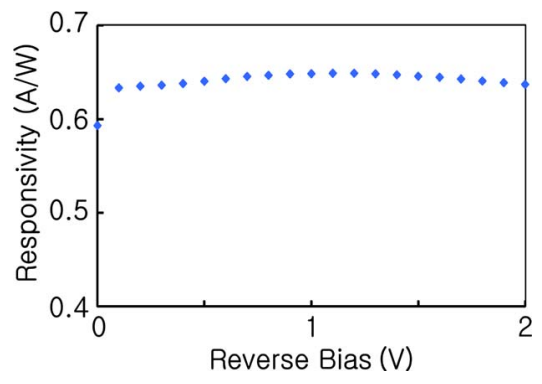

Fig. 3. Photodetector responsivity at $\lambda=1.55 \mu \mathrm{m}$ versus reverse bias for the mesa Ge p-i-n photodiodes with a radius of $100 \mu \mathrm{m}$.

For the diode operating at $1-\mathrm{V}$ reverse bias, the extracted $J_{\text {bulk }}$ and $J_{\text {surf }}$ are shown to be $3.2 \mathrm{~mA} / \mathrm{cm}^{2}$ and $62 \mu \mathrm{A} / \mathrm{cm}$, respectively. This very low bulk current density of $3.2 \mathrm{~mA} / \mathrm{cm}^{2}$ confirms the excellent Ge crystal quality and the shallow and abrupt $\mathrm{n}^{+}$junction in Ge with a high level of activation of n-type dopant, using in situ phosphorus doping during the epitaxial growth [13], [14]. This is one of lowest reported dark current density values among the Ge $p-i-n$ photodiodes [2], [3], [15]-[17].

Fig. 3 shows the responsivity $(\Re)$ versus reverse bias $(V)$ for the Ge $p-i-n$ photodiode operating at $1.55 \mu \mathrm{m}$ with $140-\mu \mathrm{W}$ incident power. The active absorption area of the device is $\pi \times 10^{4} \mu \mathrm{m}^{2}$, and the thickness of the absorbing Ge layer is $1 \mu \mathrm{m}$. A responsivity of $\sim 0.64 \mathrm{~A} / \mathrm{W}$ was measured for a reverse bias of $1 \mathrm{~V}$, corresponding to $53.6 \%$ external quantum efficiency $(\eta)$. The residual strain in this selectively grown $\mathrm{Ge}$ is $0.141 \%$, as determined by a Raman spectra measurement, while that of the bulk grown Ge is $0.204 \%$. The extracted tensile strains in both cases arise from the difference in thermal expansion coefficients between Ge and Si. During the cooling stage after Ge deposition, the decrease in the lattice constant of $\mathrm{Ge}$ is suppressed by that of the $\mathrm{Si}$ substrate, generating residual tensile strain in Ge layer [18]. The lower tensile strain value in the selectively grown Ge compared to bulk grown $\mathrm{Ge}$ can be explained by the Ge confinement by $\mathrm{SiO}_{2}$, which has a compressive strain. However, a more detailed study will be necessary to verify this effect. This $0.141 \%$ tensile strain reduces the direct bandgap of $\mathrm{Ge}$ from 0.801 to $0.781 \mathrm{eV}$, extending the effective photodetection wavelength. The responsivity of the $p-i-n$ Ge photodiode is further improved on the bulk grown Ge because of the higher tensile strain of the bulk grown Ge. This responsivity of $0.67 \mathrm{~A} / \mathrm{W}$ at $1.55 \mu \mathrm{m}$ is the highest value among the previously reported selective-area-grown $\mathrm{Ge} p-i-n$ photodiodes [15], [19]. The responsivity at $0-\mathrm{V}$ bias is also about $93 \%$ of the maximum responsivity measured, indicating excellent carrier collection efficiency [20].

From the responsivity, the absorption coefficient $(\alpha)$ of the tensile-strained Ge can be derived. The responsivity $(\Re)$ and reflectivity $(\gamma)$ of the device can be related to $\alpha$ at a certain photon energy by

$$
\alpha=-\frac{1}{t_{\mathrm{Ge}}} \ln \left(1-E \bullet \frac{\Re}{(1-r)}\right)
$$

where $t_{\mathrm{Ge}}=1 \mu \mathrm{m}$ is the thickness of undoped Ge film, and $E$ is the photon energy. Fig. 4 shows the extracted absorption 


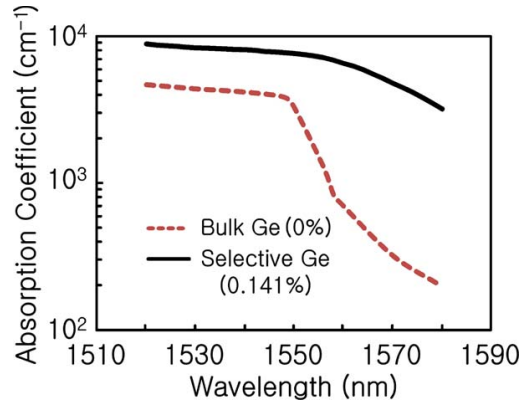

Fig. 4. Absorption coefficient at 2-V reverse-bias versus photon wavelength. The absorption coefficient $(\alpha)$ is extracted from the measured responsivity, assuming $90 \%$ internal efficiency. The $\alpha$ for bulk Ge is plotted for reference.

coefficient values versus wavelength. For comparison, the absorption curve of the unstrained Ge photodiodes is also included. The spectral responsivity shows that the optical characteristics of the selectively grown Ge layer is affected by the residual tensile strain [18]. It is clear that the absorption edge of strained Ge has shifted toward longer wavelengths. Spectral measurement verified the red shift of the absorption edge corresponding to $0.141 \%$ tensile strain, which leads to an enhanced absorption efficiency. The increase in absorption coefficients greatly improves the responsivity of the Ge photodiodes on the selectively grown Ge for monolithic integration on Si. Moreover, MHAH-Ge layers can absorb the same amount of light intensity in thinner layers, owing to the high absorption coefficient. For instance, at $\lambda=1550 \mathrm{~nm}$, the intrinsic layer could be made half the thickness compared to that of bulk-Ge detectors, resulting in shorter transit times for carrier collection, hence higher speed of operation.

\section{CONCLUSION}

We have demonstrated a $0.14 \%$ tensile-strained normal incidence $\mathrm{Ge} p-i-n$ photodiode selectively grown on $\mathrm{Si}$ for monolithic integration. A low bulk dark current density of $3.2 \mathrm{~mA} / \mathrm{cm}^{2}$ indicates good Ge film quality and a highly activated in situ doped $\mathrm{n}^{+}$layer. Responsivities of $0.64 \mathrm{~A} / \mathrm{W}$ were achieved at $1.55 \mu \mathrm{m}$. The $0.14 \%$ residual tensile strain in the selectively grown Ge resulted in an enhanced efficiency in the near infrared regime and shifted the absorption edge to longer wavelengths. This high efficiency even at low reverse bias makes this technology a promising candidate for monolithic integration of $\mathrm{Ge}$ optoelectronics on Si for optical communication.

\section{REFERENCES}

[1] S. Luryi, A. Kastalsky, and J. C. Bean, "New infrared detector on a silicon chip," IEEE Trans. Electron Devices, vol. ED-31, no. 9, pp. 1135-1139, Sep. 1984.

[2] J. Liu, D. D. Cannon, K. Wada, Y. Ishikawa, S. Jongthammanurak, D. T. Danielson, J. Michel, and L. C. Kimerling, "Tensile strained Ge p-i-n photodetectors on Si platform for C and L band telecommunications," Appl. Phys. Lett., vol. 87, no. 1, p. 011110, Jul. 2005.
[3] M. Jutzi, M. Berroth, G. Wohl, M. Oehme, and E. Kasper, "Ge-on-Si vertical incidence photodiodes with 39-GHz bandwidth," IEEE Photon. Technol. Lett., vol. 17, no. 7, pp. 1510-1512, Jul. 2005.

[4] T. Yin, R. Cohen, M. M. Morse, G. Sarid, Y. Chetrit, D. Rubin, and M. J. Paniccia, "31 GHz Ge n-i-p waveguide photodetectors on siliconon-insulator substrate," Opt. Express, vol. 15, no. 21, pp. 13965-13971, Oct. 2007.

[5] L. Vivien, J. Osmond, J. Fedeli, D. Marris-Morini, P. Crozat, J. Damlencourt, E. Cassan, Y. Lecunff, and S. Laval, " $42 \mathrm{GHz}$ p.i.n germanium photodetector integrated in a silicon-on-insulator waveguide," Opt. Express, vol. 17, no. 8, pp. 6252-6257, Apr. 2009.

[6] J. M. Hartman, J.-F. Damlencourt, Y. Bogumilowicz, P. Holliger, G. Rolland, and T. Billon, "Reduced pressure-chemical vapor deposition of intrinsic and doped Ge layers on $\mathrm{Si}(001)$ for microelectronics and optoelectronics purposes," J. Cryst. Growth, vol. 274, no. 1/2, p. 90, 2005.

[7] H. Yu, S.-L. Cheng, P. B. Griffin, Y. Nishi, and K. C. Saraswat, "Germanium in situ doped epitaxial growth on $\mathrm{Si}$ for high-performance $\mathrm{n}^{+} \mathrm{p}$-junction diode," IEEE Electron Devices Lett., vol. 30, no. 9, pp. $1002-1004$, Sep. 2009.

[8] C. O. Chui, K. Gopalakrishnan, P. B. Griffin, J. D. Plummer, and K. C. Saraswat, "Activation and diffusion studies of ion-implanted $\mathrm{p}$ and $\mathrm{n}$ dopants in germanium," Appl. Phys. Lett., vol. 83, no. 16, pp. 3275-3277, Oct. 2003.

[9] W. C. Dunlap, "Diffusion of impurities in germanium," Phys. Rev., vol. 94, no. 6, pp. 1531-1540, Jun. 1954.

[10] A. Nayfeh, C. O. Chui, K. C. Saraswat, and T. Yonehara, "Effects of hydrogen annealing on heteroepitaxial-Ge layers on Si: Surface roughness and electrical quality," Appl. Phys. Lett., vol. 85, no. 14, p. 2815, Oct. 2004.

[11] L. M. Giovane, H. C. Luan, A. M. Agarwal, and L. C. Kimerling, "Correlation between leakage current density and threading dislocation density in SiGe p-i-n diodes grown on relaxed graded buffer layers," Appl. Phys. Lett., vol. 78, no. 4, p. 541, Jan. 2001.

[12] J. D. Schaub, R. Li, S. M. Csutak, and J. C. Campbell, "High-speed monolithic silicon photoreceivers on high resistivity and SOI substrates," J. Lightwave Technol., vol. 19, no. 2, pp. 272-278, Feb. 2001.

[13] A. K. Okyay, A. Nayfeh, N. Ozguven, T. Yonehara, P. C. McIntyre, and K. C. Saraswat, "Strain enhanced high efficiency germanium photodetectors in the near infrared for integration with Si," in Proc. IEEE LEOS, 2006, pp. 460-461.

[14] A. K. Okyay, A. Nayfeh, T. Yonehara, A. Marshall, P. C. McIntyre, and K. C. Saraswat, "High-efficiency metal-semiconductor-metal photodetectors on heteroepitaxially grown $\mathrm{Ge}$ on $\mathrm{Si}$," Opt. Lett., vol. 31, no. 17, pp. 2565-2567, Sep. 2006.

[15] T. H. Loh, H. S. Nguyen, R. Murthy, M. B. Yu, W. Y. Loh, G. Q. Lo, N. Balasubramanian, and D. L. Kwong, "Selective epitaxial germanium on silicon-on-insulator high speed photodetectors using low-temperature ultrathin $\mathrm{Si}_{0.8} \mathrm{Ge}_{0.2}$ buffer," Appl. Phys. Lett., vol. 91, no. 7, p. 073503 , Aug. 2007.

[16] S. Fama, L. Colace, G. Masini, and G. Assanto, "High performance germanium-on-silicon detectors for optical communications," Appl. Phys. Lett., vol. 81, no. 4, pp. 586-588, Jul. 2002.

[17] Z. Huang, J. Oh, and J. C. Campbell, "Back-side-illuminated high-speed Ge photodetector fabricated on Si substrate using thin SiGe buffer layers," Appl. Phys. Lett., vol. 85, no. 15, p. 3286, Oct. 2004.

[18] Y. Ishikawa, K. Wada, D. D. Cannon, J. Liu, H. C. Luan, and L. C. Kimerling, "Strain-induced band gap shrinkage in Ge grown on Si substrate," Appl. Phys. Lett., vol. 82, no. 13, p. 2044, Mar. 2003.

[19] S. Park, Y. Ishikawa, T. Tsuchizawa, T. Watanabe, K. Yamada, S. Itabashi, and K. Wada, "Spectral responsivity of vertical p-i-n photodiode of selectively grown Ge on silicon-on-insulator platform," in Proc. IEEE Int. Conf. Group IV Photon., 2007, pp. 55-57.

[20] L. Colace, G. Masini, G. Assanto, H. C. Luan, K. Wada, and L. C. Kimerling, "Efficient high-speed near-infrared Ge photodetectors integrated on Si substrates," Appl. Phys. Lett., vol. 76, no. 10, p. 1231, Mar. 2000. 